\title{
SIMULATION STUDIES ON THE RADIOFREQUENCY GUN SATURATED EMISSION
}

\author{
Sergey M. Polozov \\ National Research Nuclear University- \\ Moscow Engineering Physics Institute \\ Russia \\ SMPolozov@mephi.ru
}

\author{
Vladimir I. Rashchikov \\ National Research Nuclear University- \\ Moscow Engineering Physics Institute \\ Russia \\ VIRashchikov@mephi.ru
}

Article history:

Received 06.09.2020, Accepted 14.09.2020

\begin{abstract}
The emission of a short high charged electron bunch in a radiofrequency gun was simulated. The numerical model is supplemented by a positive charge arising and dynamically changing during emission in the cathode region. The numerical results are compared with experimental data.
\end{abstract}

\section{Key words}

Photogun, beam dynamics, longitudinal and transverse motion stability.

\section{Introduction}

High brightness electron sources are key components necessary for the successful operation of modern free electron lasers, new sources of synchrotron radiation and lepton colliders. For such installations, it is necessary to have a rather high bunch charge ( $\sim$ units $\mathrm{nC})$, a very small transverse normalized emittance $(<1 \mathrm{~mm} \mathrm{mrad})$, rather short bunches $(\sim 1-10 \mathrm{ps})$, and a small energy spread $(<1 \%)$ after radiofrequency (RF) gun. For example, for a European X-ray Free Electron Laser (European XFEL) photo gun, electron bunches with a charge of $1 \mathrm{nC} /$ bunch and a normalized transverse emittance $<0.9 \mathrm{~mm}$ mrad should be generated by an RF gun operating on a $\mathrm{Cs}_{2} \mathrm{Te}$ photocathode with a high electric field on the cathode surface $(\sim 60 \mathrm{MV} / \mathrm{m})$ and repetition rate up to 27,000 pulses per second. For the lepton version of the CERN Future Circular Collider (CERN FCC-ee), it is necessary to create bunches with a charge of up to $\sim 6 \mathrm{nC}$ with a pulse duration of $\sim 1 \mathrm{ps}$ for injection and conversion of electrons into positrons. Detailed studies of the photoemission process are crucial for understanding the beam dynamics with the predominant influence of the space charge in photo injectors, without which it is difficult to achieve the above results.

\section{Simulation Model and Results}

Existing models of photoemission from metal and semiconductor photocathodes are associated with "single-particle" emission, which neglects collective effects. To bring theoretical understanding closer to experimental observation, one should take into account these collective effects at all stages of photoemission, as well as nonlinear effects that affect beam formation.

The aim of this work is to create a photo injection model in the space charge dominated regime and at presence of strong electromagnetic fields. The developed model is based on 2.5 dimensional finite difference-time domain (FDTD) particle-in-cell (PIC) code SUMA [Rashchikov, 1990]. This code has been tested and widely used to model various physical processes [Rashchikov and Didenko, 1991; Rashchikov et al., 2011; Polozov and Rashchikov, 2018; Rashchikov and Shikanov, 2019]. Part of the code responsible for the charged particles emission was rewritten according to our understanding of the physical process.

For comparison, we used the results obtained in the Photo Injector Test Facility at DESY, Zeuthen (PITZ) both in the computer simulation and in experiment [Krasilnikov et al., 2012; Chen et al., 2018; Krasilnikov et al., 2019; Li et al., 2019]. We simulated only first 0.6 cell of PITZ L-band normal conducting 1.6 cell RF gun with a $\mathrm{Cs}_{2} \mathrm{Te}$ photocathode (Fig. 1).

The photocathode will be arranged in 0.6 cell's sidewall, therefore accelerating field on the sidewall's surface must be as high as possible. That is the reason of making 0.6 cell length. The gun consists of a 1.3 $\mathrm{GHz}$ copper cavity operated in $\pi$-mode with RF field $60 \mathrm{MV} / \mathrm{m}$ on the cathode and 42 degrease optimal phase with respect to the bunch head. The laser pulse temporal profile was chosen as the flattop one with $21.5 \mathrm{ps}$ FWHM (full width at half maximum) and rise/fall times 


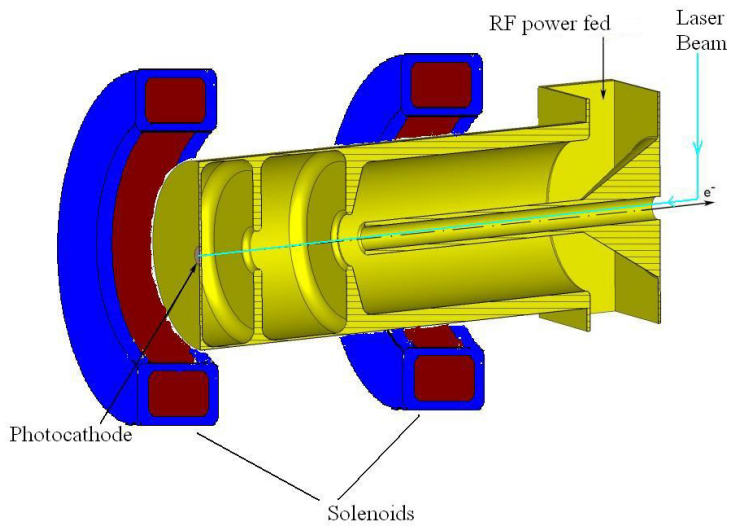

Figure 1. The general scheme of PITZ RF gun

of $\sim 2$ ps. Transverse beam space charge distribution as and at [Li et al., 2019] was chosen as uniform inside the radius of beam shaping aperture and then decreased with radius according to the Gauss law.

The analysis of discrepancies between the results of calculations and experimental data, mainly based on the results obtained in PITZ, as well as taking into account the extensive experience of previous calculations of electron and ion sources, allowed us to draw the following conclusions. The discrepancies in measured and simulated emission curves - extracted from the photocathode charge versus photocathode laser pulse energy (input charge) Fig. 2, might be easily explained by appearance of positive charge near the cathode surface during the emission process. The explanation for this appearance may be as follows.

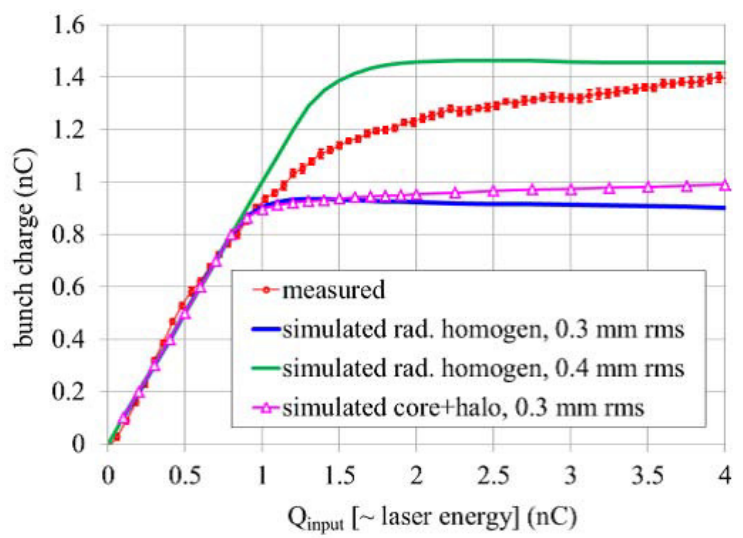

Figure 2. Dependence of the accelerated bunch charge on the photocathode laser pulse energy, measured and simulated for various transverse laser distributions (Fig. 5 from [Krasilnikov et al., 2019])

Firstly, the electrons, which arise in the surface layer of the photocathode semiconductor due to the energy of the laser pulse, are quickly removed by a strong RF field that has penetrated the surface layer, the depth of which is less than the skin layer. With such field gradients, the rate of electrons exiting from the surface layer of the laser-irradiated region of the semiconductor can significantly exceed their inflow. This leads to the appearance of a positive charge, the magnitude of which is proportional to the magnitude of the emitted current, and is determined by the difference in the rates of exit and influx of electrons into the studied region.

Secondly, ions are generated through electron impact ionization of residual gas [Qiang, 2010]. Taking into account the energy value, at which the maximum ionization cross section is reached $(\sim 50-100 \mathrm{eV})$ and slow motion of ions, this may be the next reason for the appearance of a positive charge in the immediate vicinity of the cathode.

Part of the code SUMA, responsible for photoemission, was modified in a way to simulate the appearance of the positive charges in the cathode region and there further dynamics. At each time step, the value of the positive charge increases in proportion to the emitted charge and decreases exponentially with a rate proportional to the rate of electron influx into the simulated region. The calculation results are presented in Fig. 3.

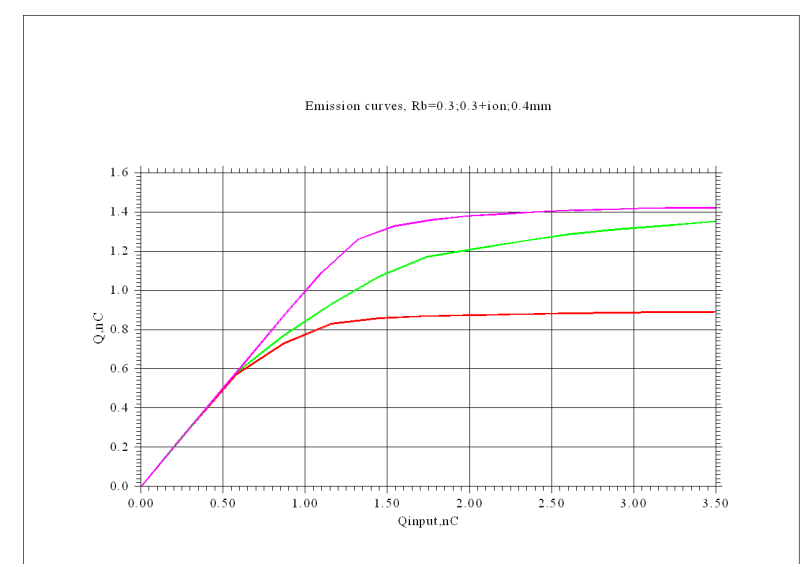

Figure 3. Figure 3. Dependence of the accelerated bunch charge on the photocathode laser pulse energy, measured and simulated for various transverse laser distributions (SUMA results). Red and magenta curves corresponds to bunch radius 0.3 and $0.4 \mathrm{~mm}$ respectively without taking into account ions fields. Green curve corresponds to bunch radius $0.3 \mathrm{~mm}$ with taking into account ions fields

Fairly good agreement is seen in the behavior of the curves on both figures. The red and magenta curves of Fig.3 represent the simulation results without taking into account the appearance of a positive charge in the cathode region ( correspond to magenta and green curves on Fig. 2). The green curve in Fig. 3 computed by improved simulation model and is in good agreement with the PITZ measurements (red curve in Fig. 2).

Fig. 4 illustrates of the emitted current pulse shape variation with bunch charge growth without taking into 


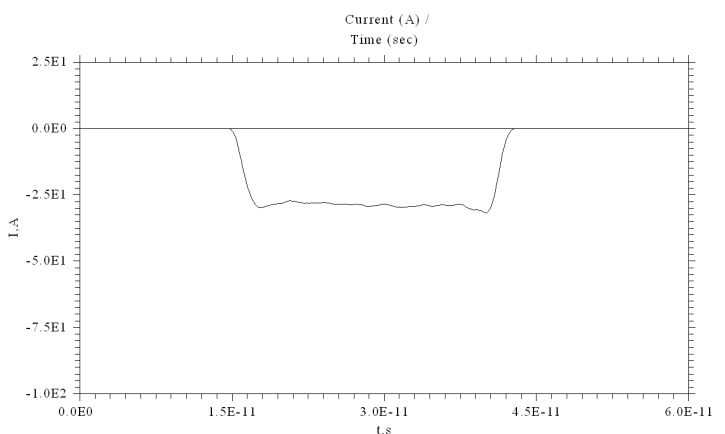

account (top three pictures) and taking into account the appearance of a positive charge in the cathode region (three bottom pictures).

It can be seen that without taking into account the influence of a positive charge in the cathode region (top three pictures), with increasing laser power, the current amplitude ceases to grow, and the shape is distorted, which is associated with the formation of a virtual cathode.

Taking into account the influence of a positive charge in the cathode region (three bottom pictures), the picture changes. With increasing laser power, the current amplitude continues to grow, and the shape is distorted slightly, which can be explained by partial compensation of the space charge forces of the emitted electrons by the field of the arising positive charge.

\section{Conclusion}

In this paper we have described the preliminary results of computer simulation of PITZ photo gun emission, using an advanced photo emission model. Comparison of the obtained results with the experimental and calculated PITZ data allows us to speak about the correctness of the assumptions introduced into the model. Nevertheless, it should be noted that this is only preliminary results and the model requires further improvement.

\section{Acknowledgements}

The reported study was funded by Russian Foundation for Basic Research (RFBR), project number 19-2912036.

\section{References}

Chen, Y. et al. (2018). Modeling and simulation of RF photoinjectors for coherent light sources. Nuclear Instruments and Methods in Physics Research A, 889, pp. 129-137.

Krasilnikov, M. et al. (2012). Experimentally minimized beam emittance from an L-band photoinjector. Phys. Rev. ST Accel. Beams, 15, 100701.

Krasilnikov, M., Chen, Y., and Stephan, F. (2019). Studies of space charge dominated electron photoemission at PITZ. J. Phys.: Conf. Ser., 1238, 012064.

Li, X.-K. et al. (2019) Simulation Studies on the Saturated Emission at PITZ. 39th Free Electron Laser Conf., FEL2019, WEP052.

Qiang, J. (2010). Particle-in-cell/Monte Carlo simulation of ion back bombardment in a high average current RF photo-gun. Nuclear Instruments and Methods in Physics Research A, 614, pp. 1-9.

Polozov, S.M. and Rashchikov, V.I. (2018). Longitudinal motion stability of electrons inside the plasma channel of LPWA. Cybernetics and Physics, 7 (4), pp. 228232.

Figure 4. Emitted current pulse shape variation with bunch charge growth ( 0.6,1.0,1.4 $\mathrm{nC}$ respectively) without (top three pictures) and 
Rashchikov, V.I. (1990). Electromagnetic field calculation in complex geometry structures. Problems of Atomic Science and Technology. Series: Nuclear Physics Investigations, 10 (18), pp. 50-53.

Rashchikov, V.I. and Didenko, A.N. (1991). High power microwave generation in virtual cathode systems. 1991 IEEE Particle Accelerator Conference, pp. 31113113.

Didenko, A.N., Rashchikov, V.I., and Fortov, V.E.
(2011). Mechanism of generation of high-intensity terahetrz radiation under the action of high-power laser pulsed on a target. Technical Physics, 56, (10), pp. $1535-1538$.

Rashchikov, V.I. and Shikanov, A.E. (2019). Compact plasma reflex triode for neutron generation. IEEE Transactions on Plasma Science, 47 (2), pp. 12791282. 